\title{
The Expression and Function of Metastases Associated Lung Adenocarcinoma Transcript-1 Long Non-Coding RNA in Subchondral Bone and Osteoblasts from Patients with Osteoarthritis
}

\author{
Fawzeyah A. Alnajjar ${ }^{1}{ }^{(D)}$, Archana Sharma-Oates ${ }^{1}$, Susanne N. Wijesinghe ${ }^{1}$, Hussein Farah ${ }^{1}$, \\ Dominika E. Nanus ${ }^{1}$, Tom Nicholson ${ }^{1}$, Edward T. Davis ${ }^{2}$ and Simon W. Jones ${ }^{1, *}$ \\ 1 Institute of Inflammation and Ageing, MRC-ARUK Centre for Musculoskeletal Ageing Research, \\ University of Birmingham, Birmingham B15 2TT, UK; fxa790@student.bham.ac.uk (F.A.A.); \\ a.sharma-oates@bham.ac.uk (A.S.-O.); s.n.wijesinghe@bham.ac.uk (S.N.W.); \\ hxf820@student.bham.ac.uk (H.F.); d.nanus@bham.ac.uk (D.E.N.); t.a.nicholson@bham.ac.uk (T.N.) \\ 2 The Royal Orthopaedic Hospital, Birmingham B31 2AP, UK; edward.davis@nhs.net \\ * Correspondence: s.w.jones@bham.ac.uk; +44-1213713224
}

Citation: Alnajjar, F.A.;

Sharma-Oates, A.; Wijesinghe, S.N.; Farah, H.; Nanus, D.E.; Nicholson, T.; Davis, E.T.; Jones, S.W. The

Expression and Function of

Metastases Associated Lung

Adenocarcinoma Transcript-1 Long Non-Coding RNA in Subchondral Bone and Osteoblasts from Patients with Osteoarthritis. Cells 2021, 10, 786. https://doi.org/10.3390/cells10040786

Academic Editor: Cord Brakebusch

Received: 2 March 2021

Accepted: 30 March 2021

Published: 1 April 2021

Publisher's Note: MDPI stays neutral with regard to jurisdictional claims in published maps and institutional affiliations.

Copyright: (c) 2021 by the authors. Licensee MDPI, Basel, Switzerland. This article is an open access article distributed under the terms and conditions of the Creative Commons Attribution (CC BY) license (https:// creativecommons.org/licenses/by/ $4.0 /)$.

\begin{abstract}
Metastasis Associated Lung Adenocarcinoma Transcript-1 (MALAT1) is implicated in regulating the inflammatory response and in the pathology of several chronic inflammatory diseases, including osteoarthritis (OA). The purpose of this study was to examine the relationship between OA subchondral bone expression of MALAT1 with parameters of joint health and biomarkers of joint inflammation, and to determine its functional role in human OA osteoblasts. Subchondral bone and blood were collected from hip and knee OA patients $(n=17)$ and bone only from neck of femur fracture patients $(n=6)$ undergoing joint replacement surgery. Cytokines were determined by multiplex assays and ELISA, and gene expression by qPCR. MALAT1 loss of function was performed in OA patient osteoblasts using locked nucleic acids. The osteoblast transcriptome was analysed by RNASeq and pathway analysis. Bone expression of MALAT1 positively correlated to serum DKK1 and galectin- 1 concentrations, and in OA patient osteoblasts was induced in response to IL-1 $\beta$ stimulation. Osteoblasts depleted of MALAT1 exhibited differential expression ( $>1.5$ fold change) of 155 genes, including PTGS2. Both basal and IL-1 $\beta$-mediated PGE2 secretion was greater in MALAT1 depleted osteoblasts. The induction of MALAT1 in human OA osteoblasts upon inflammatory challenge and its modulation of PGE2 production suggests that MALAT1 may play a role in regulating inflammation in OA subchondral bone.
\end{abstract}

Keywords: osteoarthritis; osteoblasts; MALAT1; long non-coding RNA

\section{Introduction}

Osteoarthritis (OA) is a degenerative joint disease and a leading cause of pain and disability for which there are currently no approved pharmacological disease modifying therapeutics [1]. Current OA treatment is limited to generic analgesics that have limited efficacy, physical therapy and ultimately joint replacement surgery [2,3]. Non-pharmacological therapeutic strategies such as platelet rich plasma and mesenchymal stem cells, aimed at promoting cartilage repair, hold promise but require further clinical studies to confirm efficacy and safety [4]. There is therefore a high unmet healthcare need to better understand the pathophysiology of OA and to develop an effective disease modifying therapeutic [5]. Importantly, despite initially considered to be a disease solely of the articular cartilage, it is now widely accepted that $\mathrm{OA}$ is a disease that encompasses the whole joint, including underlying subchondral bone tissue [2]. Pathological changes to OA subchondral bone tissue, characterised by trabecular thickening [6], abnormal type I Collagen production $[7,8]$ and the formation of osteophytes, occurs early in the disease course of OA [9] and involves 
changes to the osteoblast phenotype [10]. Furthermore, in animals that are prone to developing OA trabecular thickening occurs prior to cartilage degeneration [11] and thus it has been suggested that this alteration to the bone architecture may pathologically alter the loading biomechanics, thus promoting cartilage degeneration [12]. Therefore, identifying novel regulators that mediate the functional phenotype of osteoblasts, the cells which regulate bone remodelling, may identify new opportunities for the development of new OA therapeutics that target the bone.

Recently, long non-coding RNAs (lncRNAs) have emerged as novel epigenetic regulators of gene transcription $[13,14]$ and of mediating several cellular processes including cellular proliferation, cell cycle control, apoptosis and the innate inflammatory response [15-18]. As such, lncRNAs have now been implicated in the pathology of several chronic inflammatory diseases [19,20], including OA [21]. Indeed, lncRNAs have been identified that are differentially expressed in OA diseased cartilage and which mediate the IL- $1 \beta$ inflammatory response in OA chondrocytes [21,22]. Furthermore, we recently reported that Metastasis Associated Lung Adenocarcinoma Transcript-1 (MALAT1) lncRNA modulates the inflammatory phenotype of synovial fibroblasts in the OA synovial joint lining by mediating the production of CXCL8 [23]. However, importantly, MALAT1 lncRNA has now emerged as a central mediator of osteoblast function and bone homeostasis. Expression of MALAT1 is reported to be greater in the bone tissue of patients who exhibit aseptic loosening following a hip replacement [24] and its upregulation has been implicated in lumber intervertebral disc degeneration [25]. In vitro, knockdown of MALAT1 has been demonstrated to inhibit the proliferation of the human osteoblast cell line hFOB 1.19 [24], and MALAT1 sponging of the microRNA miR-30 has been shown to promote the osteoblast differentiation of mesenchymal stem cells by inducing RUNX2 expression [26]. Furthermore, MALAT1 is associated with abnormal osteogenic and adipogenic differentiation of BMSCs in the patients with osteonecrosis of the femoral head [27].

These data suggest that MALAT1 could play a key role in the pathological changes that occur in OA diseased bone. However, currently the expression and functional role of MALAT1 in OA subchondral bone has not been reported. Therefore, the aim of this study was to profile the expression of MALAT1 in the subchondral bone tissue of patients with either knee or hip OA and its relationship to parameters of joint damage and to examine the functional role of MALAT1 in OA patient primary osteoblasts.

\section{Materials and Methods}

\subsection{Patient Recruitment}

Following ethical approval (UK NRES 16/SS/0172 \& NRES 14/ES/1044 approved on 31 October 2016 and 12 August 2014, respectively), subchondral bone tissue and blood was collected from a total of 17 patients ( 9 males, 8 females) with end-stage OA comprising of 9 patients with hip OA and 8 patients with knee OA and from neck of femur fracture (NOF\#) patients without OA $(n=6)$, who were undergoing surgery at Russell's Hall Hospital (Dudley, UK) or the Royal Orthopaedic Hospital (Birmingham UK). OA patient characteristics are detailed in Table 1. All participants demonstrating secondary causes of OA, such as avascular necrosis, Perthes disease, developmental dysplasia, previous acetabular or femoral neck fractures and slipped upper femoral epiphysis, were excluded from the study. Informed consent was obtained from all subjects involved in the study. 
Table 1. OA patient characteristics.

\begin{tabular}{cccc}
\hline & All OA Patients & Knee OA Patients & Hip OA Patients \\
\hline Age (years) & $65 \pm 2.2$ & $67 \pm 3.1$ & $63.2 \pm 3.2$ \\
Gender $(\mathrm{m}: \mathrm{f})$ & $9: 7$ & $4: 4$ & $5: 3$ \\
Height $(\mathrm{cm})$ & $167.2 \pm 3.2$ & $164.8 \pm 5.4$ & $169.4 \pm 3.8$ \\
Weight $(\mathrm{Kg})$ & $82.4 \pm 4.2$ & $84.2 \pm 7.9$ & $80.8 \pm 4.3$ \\
BMI $\left(\mathrm{kg} / \mathrm{m}^{2}\right)$ & $29.7 \pm 1.6$ & $31.1 \pm 2.7$ & $28.4 \pm 1.9$ \\
\% Fat & $34.8 \pm 2.4$ & $35.0 \pm 5.1$ & $34.8 \pm 2.3$ \\
WHR 1 & $0.92 \pm 0.02$ & $0.93 \pm 0.03$ & $0.91 \pm 0.03$ \\
KL Grade & $4(3.5-4)$ & $4(3.25-4)$ & $4(3.5-4)$ \\
\hline
\end{tabular}

${ }^{1}$ WHR = Ratio of waist to hip circumference. All values represent mean \pm SEM, except KL grade which is shown as median (25th-75th percentile).

\subsection{Isolation and Culture of Primary Osteoblasts}

Primary OA osteoblasts were cultured from OA subchondral bone tissue. The bone was cut into small pieces approximately $2 \mathrm{~mm}^{3}$ and washed 3 times in $30 \mathrm{~mL}$ of complete osteoblast culture media (DMEM, 10\% FBS, 100 Units $/ \mathrm{mL}$ Penicillin, $100 \mathrm{ug} / \mathrm{mL}$ Streptomycin, 1\% Non-essential amino acids, L-glutamine $2 \mathrm{mM}$, ascorbic acid $50 \mathrm{ug} / \mathrm{mL}$, Dexamethasone $10 \mathrm{nM}$, and $\beta$-glycerophosphate $2 \mathrm{mM}$ ) to remove excess fat. The bone chips were then placed in a T75 flasks with fresh culture media and incubated in at $37^{\circ} \mathrm{C}$ in a humidified atmosphere containing $5 \% \mathrm{CO}_{2}$. The media was changed every $3-4$ days, and the chips were removed after 7-14 days when the osteoblasts outgrowth occurred.

\subsection{Analysis of Serum Cytokines}

The serum concentration of 24 cytokines were determined using Luminex multiplex platform (Luminex R\&D systems) according to the manufacturer's instructions, having been diluted to 1:2 in assay buffer.

\subsection{MALAT1 Functional Studies in Human OA Osteoblasts}

In vitro loss of function studies were performed using lipofectamine 3000 (Qiagen, Manchester, UK) to transfect primary osteoblasts with two different Locked nucleic acids (LNAs) targeting MALAT1 or with a control (NC) LNA. To determine the effect of MALAT1 knockdown on the OA osteoblast transcriptome, total RNA was extracted (RNeasy columns, Qiagen Manchester UK) $24 \mathrm{~h}$ following transfection with LNAs. RNA integrity (RIN) was evaluated (Agilent Bioanalyser, Cheshire UK) with a RIN of $>7$ deemed of sufficient quality for RNA sequencing analysis using the QuantSeq $3^{\prime}$ kit (Lexogen, Vienna, Austria). The sequenced reads were mapped to the hg38 reference human genome using Star Aligner version 2.5.2b [28]. Differential gene expression analysis and $\log 2$ fold changes were computed using DESeq2 version 1.26.0 [29].

The effect of MALAT1 knockdown on the production of PGE2 and osteoprotegrin (OPG) was determined by ELISA according to the manufacturer's instructions (RnD systems, Oxford, UK). Alkaline phosphatase (ALP) activity was quantified in osteoblast lysates prepared in RIPA buffer diluted 1:5 with $1 \mathrm{mM} \mathrm{MgCl} 2$. In brief, diluted osteoblast lysates were combined with ALP substrate (Sigma, Gillingham, UK) and incubated at $37^{\circ} \mathrm{C}$ for $15 \mathrm{~min}$. The reaction was stopped with the addition of $0.1 \mathrm{~N} \mathrm{NaOH}$ and absorbance read at $405 \mathrm{~nm}$ on a microplate reader. The degree of osteoblast mineralisation was quantified by staining of mineralised nodules using an alizarin red solution (0.5\% Alizarin Red in $1 \%$ ammonia hydroxide at $\mathrm{pH}$ 4.5; Sigma, Gillingham, UK). Following 10 min incubation at room temperature, cells were washed with PBS to remove excess stain, and then incubated in $10 \%$ cetyl pyridinium chloride (Sigma, Gillingham, UK) for $10 \mathrm{~min}$. The supernatant was then collected, diluted 1:10 with the $10 \%$ cetyl pyridinium chloride and absorbance read at OD550 $\mathrm{nm}$ on a microplate reader. 


\subsection{Pathway Analysis}

Pathway analysis was performed using the software Ingenuity Pathway Analysis (IPA; www.ingenuity.com, accessed on 31 January 2020). Differentially expressed genes (fold change of $\pm>1.5, p<0.05$ ) were analysed using a core functional analysis to identify significant canonical pathways and cellular processes.

\subsection{Statistical Analysis}

All statistics were performed using Graph Pad Prism 9.0. ANOVA followed by post hoc analysis of Bonferroni was used to test statistical significance where appropriate. Pearson correlations were performed to test the strength of association between two variables. $p$-value $<0.05$ was considered as significant.

\section{Results}

3.1. Expression of MALAT1 in OA Subchondral Bone and Relationship to Patient Characteristics and $O A$ Disease Severity

MALAT1 was highly expressed in OA subchondral bone tissue, with on average greater expression in hip OA compared to non-OA hip (NOF\#). However, this did not reach significance and there was no significant difference in expression between patients with knee OA and those with hip OA (Figure 1A). Furthermore, although on average there was greater MALAT1 expression in OA patients with greater disease severity (KL4 and joint space $<1 \mathrm{~mm}$ ), compared to those patients with KL grade 3 and joint space $>1 \mathrm{~mm}$, there was no significant relationship between MALAT1 expression and disease severity (Figure 1B; Supplementary Table S1).

Next, we examined whether the subchondral bone expression of MALAT1 was related to biomarkers of inflammation by measuring the concentration of a panel of 22 cytokines in OA patient serum by Luminex. There were significant positive correlations between MALAT1 expression and the serum concentration of both the Wnt pathway inhibitor DKK1 $\left(\mathrm{r}^{2}=0.3, p=0.04\right)$ and galectin- $1\left(\mathrm{r}^{2}=0.44, p=0.009\right)$ (Figure 1C; Table 2). However, it should be noted that these findings were not significant upon correcting for multiple comparisons (FDR), and therefore a type I error cannot be ruled out.

Table 2. Relationship between MALAT1 subchondral bone expression and the concentration of cytokines in OA patient serum.

\begin{tabular}{|c|c|c|c|}
\hline & Concentration $^{a}$ & $\mathbf{r}^{2}$ & $p$-Value \\
\hline Visfatin & $2979 \pm 355$ & 0.11 & 0.23 \\
\hline Resistin & $15441 \pm 1394$ & 0.17 & 0.18 \\
\hline Leptin & $18365 \pm 5865$ & 0.03 & 0.55 \\
\hline Adiponectin & $10640 \pm 2081^{b}$ & $<0.01$ & 0.94 \\
\hline Chemerin & $6920 \pm 937$ & $<0.01$ & 0.67 \\
\hline Dkk1 & $3.7 \pm 0.3^{b}$ & 0.3 & 0.04 \\
\hline Galectin1 & $41 \pm 3^{b}$ & 0.44 & 0.009 \\
\hline Eotaxin & $170 \pm 44$ & 0.15 & 0.16 \\
\hline Amphiregulin & $570 \pm 54$ & 0.24 & 0.07 \\
\hline Aggrecan & $183 \pm 54$ & 0.10 & 0.28 \\
\hline FABP4 & $47.4 \pm 15.2^{b}$ & 0.04 & 0.48 \\
\hline Serpin E1 & $131 \pm 19^{b}$ & 0.02 & 0.63 \\
\hline IP10 & $32.3 \pm 5.9$ & 0.03 & 0.56 \\
\hline MIP1a & $400 \pm 76$ & 0.06 & 0.39 \\
\hline MIP1b & $188 \pm 31$ & 0.01 & 0.74 \\
\hline MCP1 & $398 \pm 38$ & $<0.01$ & 0.94 \\
\hline MIP3a & $37.3 \pm 6.8$ & 0.07 & 0.38 \\
\hline IL-1 $\beta$ & $17.6 \pm 3.1$ & 0.007 & 0.78 \\
\hline IL6 & $10.9 \pm 4.1$ & 0.05 & 0.55 \\
\hline IL7 & $3.4 \pm 0.3$ & 0.09 & 0.29 \\
\hline IL10 & $4.5 \pm 0.3$ & 0.01 & 0.69 \\
\hline IL15 & $5.3 \pm 1.6$ & 0.02 & 0.66 \\
\hline $\mathrm{TNF}-\alpha$ & $4.3 \pm 0.3$ & 0.08 & 0.32 \\
\hline Gp130 & $94 \pm 7.1^{b}$ & 0.02 & 0.63 \\
\hline
\end{tabular}

a Concentration of cytokines in $\mathrm{pg} / \mathrm{mL} \pm$ SEM. Except ${ }^{\mathrm{b}}$, concentration of cytokines in $\mathrm{ng} / \mathrm{mL}$. 
A

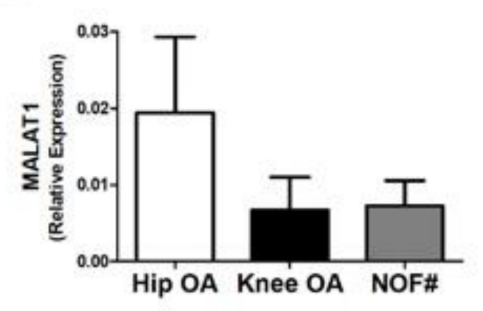

B
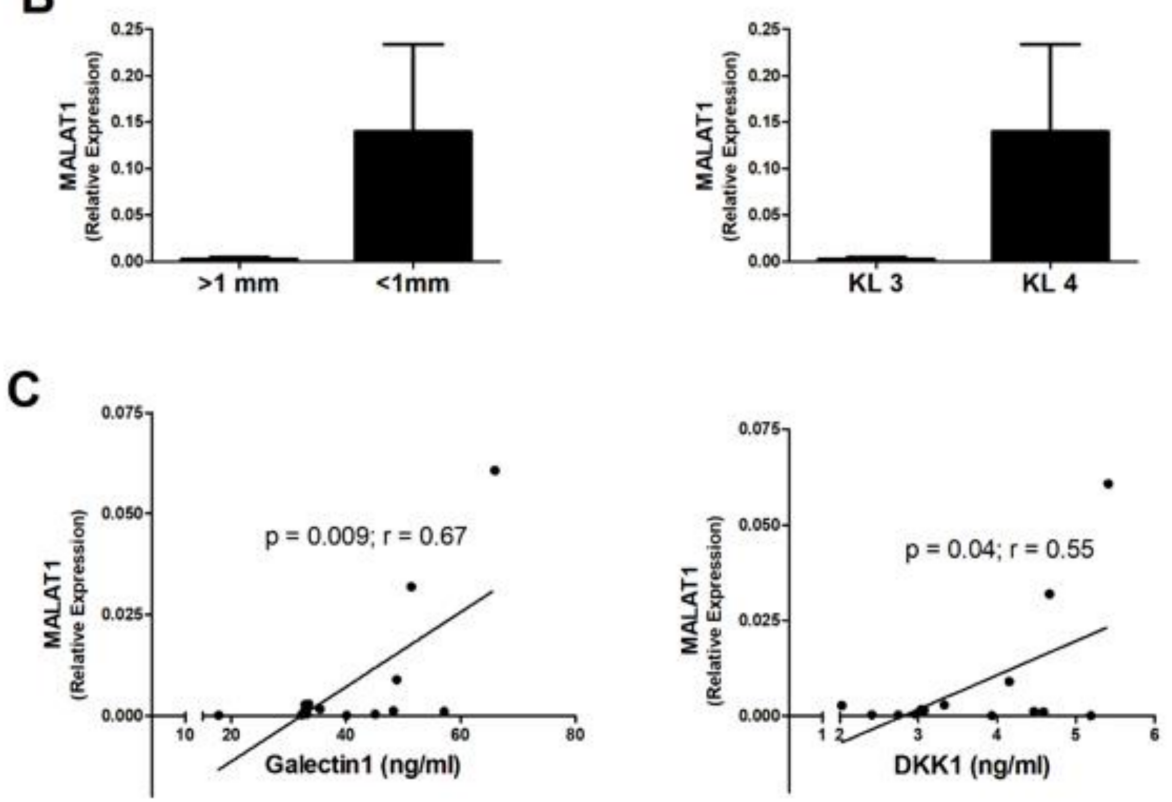

D
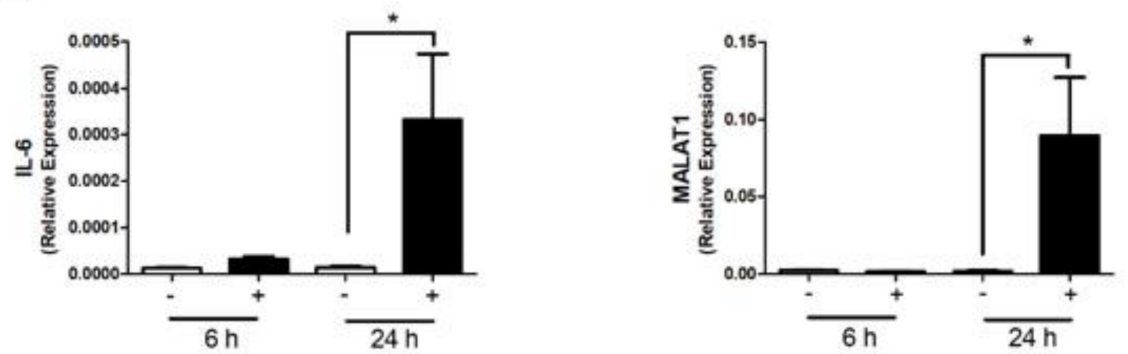

Figure 1. Expression of MALAT1 in subchondral bone and OA osteoblasts. (A) Relative expression of MALAT1 in the subchondral bone tissue of end-stage hip OA patients $(n=9)$ compared to end-stage knee OA patients $(n=8)$ and non-OA neck of femur fracture (NOF\#) patients $(n=6)$. MALAT1 expression was determined by qPCR and normalised to $18 \mathrm{~S}$. Bars represent mean expression \pm SEM. (B) Relative expression of MALAT1 in the subchondral bone tissue between OA patients with more severe radiographic signs of OA $(n=13)$ with joint space $<1 \mathrm{~mm}$ and $\mathrm{KL}$ grade 4 , compared to those with less severe joint damage $(n=4)$ with joint space $>1 \mathrm{~mm}$ and KL grade 3. (C) Correlation between subchondral bone expression of MALAT1 and the serum concentration of Galectin $1(\mathrm{ng} / \mathrm{mL})$ and DKK1 $(\mathrm{ng} / \mathrm{mL})$ in OA patients $(n=17)$. (D) Effect of IL-1 $\beta$ stimulation $(1 \mathrm{ng} / \mathrm{mL})$ upon the expression of $I L-6$ and MALAT1 in primary OA osteoblasts at $6 \mathrm{~h}$ and $24 \mathrm{~h} .{ }^{*}=p<0.05$, compared to non-stimulated control. 


\subsection{MALAT Expression in OA Osteoblasts Is Induced by Inflammatory Challenge}

Next, using an in vitro model of primary OA patient osteoblasts, we examined whether the expression of MALAT1 was induced upon an inflammatory challenge. To this end, primary osteoblasts from $n=3$ OA patients were stimulated for either $6 \mathrm{~h}$ or $24 \mathrm{~h}$ with IL-1 $\beta$ ( $1 \mathrm{ng} / \mathrm{mL}$ ), and the expression of IL-6 and MALAT1 determined by qPCR. As expected, stimulation of osteoblasts with IL- $1 \beta$ induced a significant increase in the expression of the pro-inflammatory cytokine IL-6 within $24 \mathrm{~h}$ ( 23 -fold, $p<0.05$ ), compared to non-stimulated control. Similarly, osteoblasts stimulated with IL-1 $\beta$ for $24 \mathrm{~h}$ exhibited significant increase in the expression of MALAT1 (4-fold, $p<0.05$ ), compared to non-stimulated control (Figure 1D).

\subsection{The Effect of MALAT1 Knockdown on the OA Osteoblast Transcriptome}

To examine the functional role of MALAT1 in OA subchondral osteoblasts we next conducted loss of function studies by performing targeted knockdown of MALAT1 expression using locked nucleic acids (LNAs) and examined the resulting osteoblast transcriptome by RNA sequencing. Primary OA osteoblasts ( $n=3$ OA patients) were transfected with either a non-targeting control LNA or one of two LNA duplexes targeting MALAT1. Following $24 \mathrm{~h}$ transfection, LNAs targeting MALAT1 induced knockdown of between $60-90 \%$ in the expression of MALAT1, compared to the control LNA (Figure 2A). RNAseq identified 155 transcripts (82 upregulated and 73 downregulated), which were differentially expressed ( $>1.5$-fold change, $p$-value $\leq 0.05)$ in osteoblasts transfected with the MALAT1 LNAs, compared to LNA control transfected cells. Of the upregulated transcripts, 80 were protein coding genes (including PTGS2) and 2 were antisense lncRNAs. Of the downregulated transcripts, 66 were protein-coding genes and 7 were lncRNAs, which comprised of 2 lincRNAs, 3 antisense, 1 sense intronic lncRNA and 1 pseudogene (Figure 2B,C, Supplementary Tables S2 and S3).

To understand the functional effect of this MALAT1 mediated change in osteoblast transcriptome we next analysed the 155 differentially expressed genes by bioinformatic pathway analysis (Ingenuity Pathway Analysis) to identify significantly affected canonical pathways and cellular processes. The most significant canonical pathways affected included phosphatidylcholine biosynthesis, fMLP signalling in neutrophils, NAD biosynthesis, eicosanoid biosynthesis and prostanoid biosynthesis. The significantly affected cellular processes included cell-cell signalling, DNA replication, cellular growth and proliferation and cellular development (Figure 2D).

\subsection{Modulation of MALAT1 Expression in Osteoblasts Induced PGE2 Secretion but Did Not} Impair Osteoblast OPG Secretion or Their Innate Ability to Mineralise

Given the finding that knockdown of MALAT1 induced the expression of PTGS2, we next examined if this was reflected in a greater production of the prostaglandin PGE2, a purported mediator of pain and inflammation in OA. To this end, OA osteoblasts were transfected with either a control LNA or one of two MALAT1 LNAs for $24 \mathrm{~h}$. Cells were then either stimulated for $24 \mathrm{~h}$ with IL-1 $\beta(1 \mathrm{ng} / \mathrm{mL})$ or left unstimulated and secretion of PGE2 quantified by ELISA. MALAT1 knockdown was confirmed by qPCR (Figure 3A). Compared to LNA control, cells depleted of MALAT1 exhibited greater basal and IL-1 $\beta$ induced PGE2 production (2-fold, $p<0.05)$ (Figure 3B). 
A

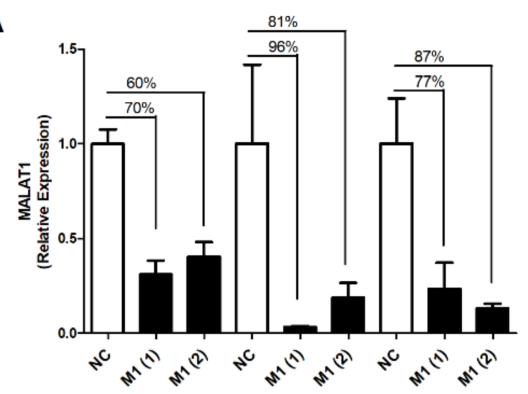

B

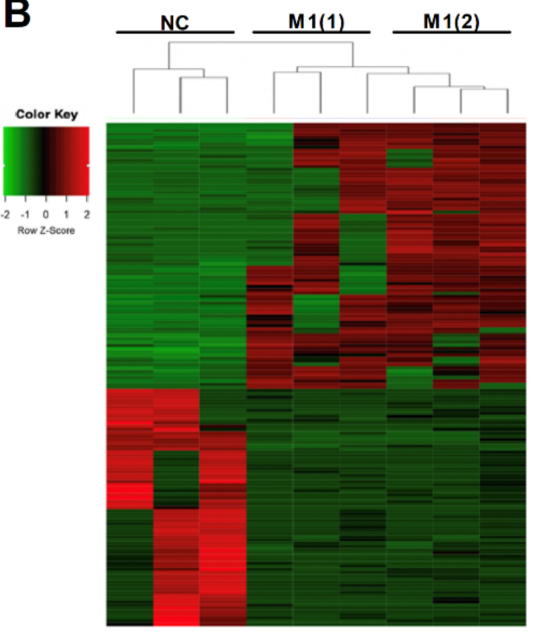

C

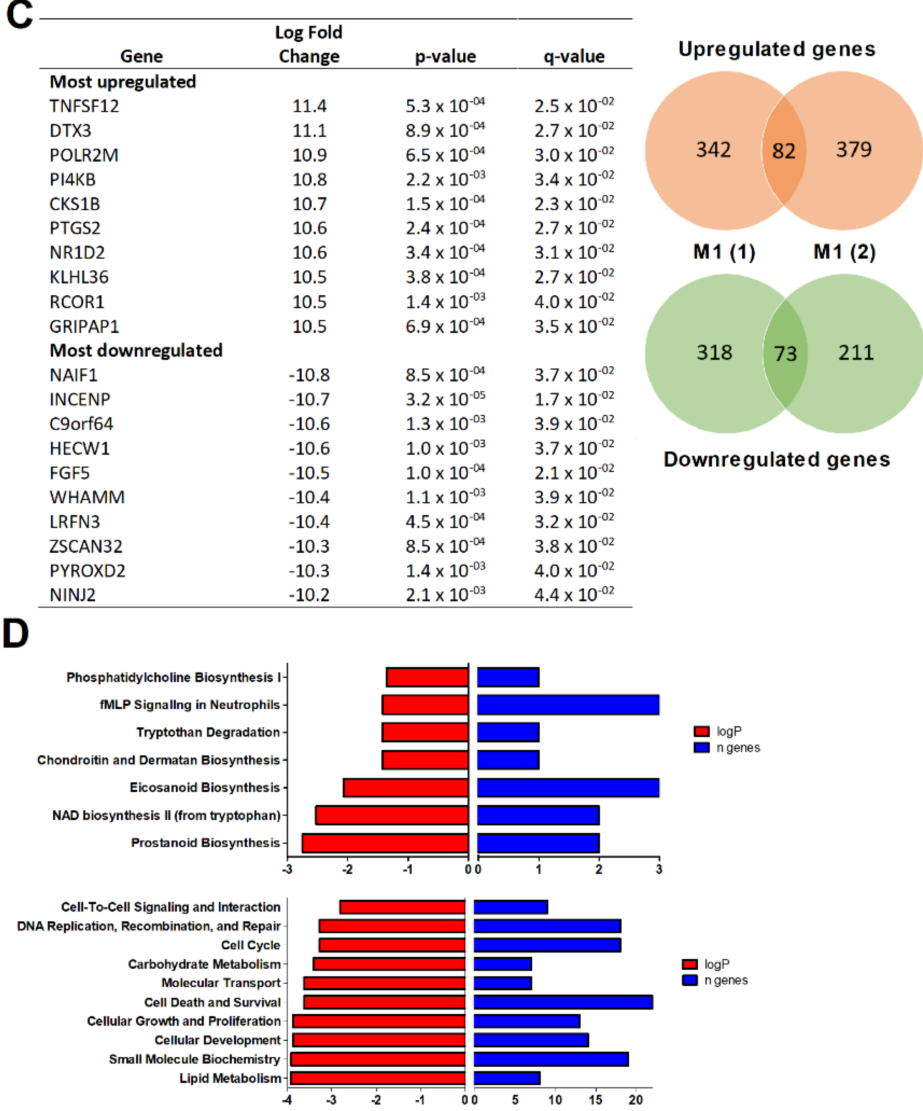

Figure 2. The effect of MALAT1 knockdown on the OA osteoblast transcriptome. (A) Knockdown of MALAT1 expression in primary OA osteoblasts. Osteoblasts were transfected with either a non-targeting control LNA (NC) or one of two LNAs targeting MALAT1, M1(1) and M1(2). Expression of MALAT1 was determined by qPCR $24 \mathrm{~h}$ after transfection and normalised to 18S. (B) Heatmap of differentially expressed genes ( $>1.5$-fold, $p<0.05)$ as determined by RNAseq (Quantseq) following $24 \mathrm{~h}$ LNA-mediated MALAT1 knockdown. (C) Table of the most upregulated and down-regulated genes following MALAT1 knockdown, with Venn diagram illustrating the total numbers of genes differentially expressed ( $>1.5$-fold, $p<0.05$ ) with each of the MALAT1 LNAs. Values represent the mean fold change between control LNA and MALAT1(1) LNA and between control LNA and MALAT1(2) LNA. (D) Top canonical pathways and cellular processes as determined by Ingenuity Pathway Analysis of the differentially expressed genes (fold change $>1.5, p<0.05$ ) following MALAT1 knockdown, with $\log P$ values representing the significance between the pathway/process and the gene dataset and $\mathrm{n}$ representing the numbers of genes within the dataset aligned to the pathway/process.

Next, we investigated whether chronic loss of function of MALAT1 would affect the innate function of osteoblasts with regard to bone remodelling by determining OPG production, alkaline phosphatase activity and osteoblast mineralisation. To this end, OA osteoblasts were transfected with either a control LNA or a MALAT1 LNA twice per week for a period of 3 weeks. During the 3-week time course, supernatants were collected to analyse OPG production by ELISA and cells lysed to measure alkaline phosphatase activity. At the end of the 3 weeks, cells were stained with alizarin red to quantify mineralisation. Twice weekly transfection with LNA maintained the significant knockdown in MALAT1 expression of between 65-85\% (Figure 3C). Production of OPG from both control and MALAT1 LNA transfected cells increased throughout the 3-week time course. However, there was no significant difference in either the rate of OPG production or in the total amount secreted (Figure 3D). Furthermore, there was no significant difference in either alkaline phosphatase activity (Figure 3E) or the degree of osteoblast mineralisation (Figure 3F) between MALAT1 depleted osteoblasts and control cells. 
A

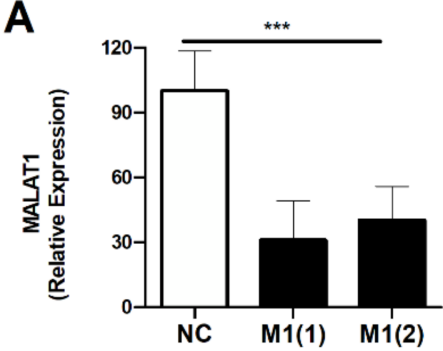

C

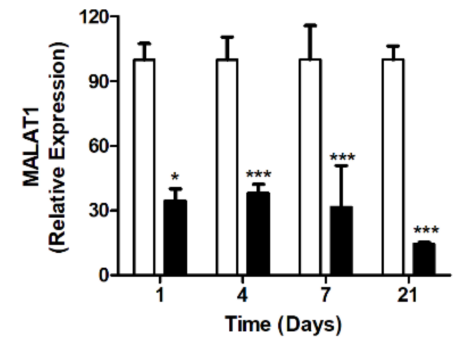

$\mathbf{F}$

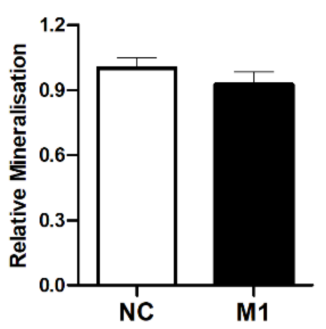

B

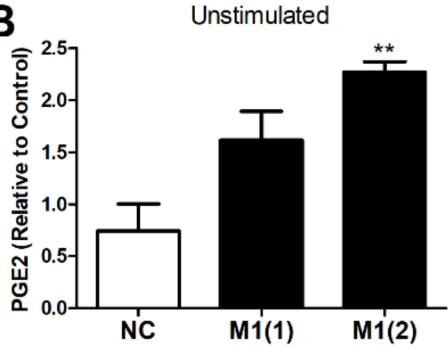

D

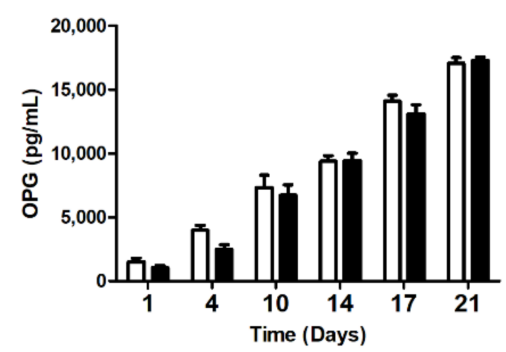

IL-1 $\beta$ Stimulated

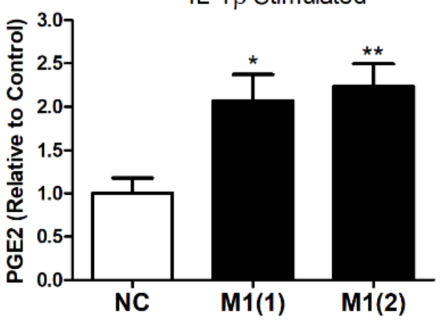

$\mathbf{E}$

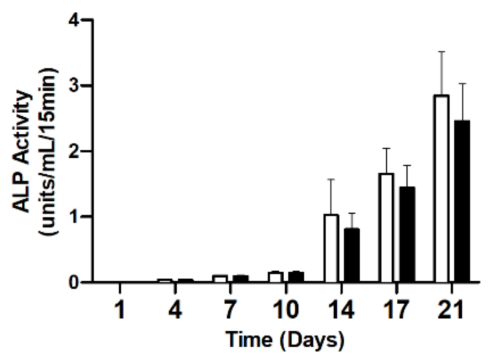

Figure 3. The effect of MALAT1 knockdown on the function of OA osteoblasts. (A) Knockdown of MALAT1 in OA osteoblasts $(n=6)$ following $24 \mathrm{~h}$ transfection with LNAs targeting MALAT1 (M1(1) and M1(2)), compared to LNA control (NC). (B) Effect of MALAT1 knockdown on $24 \mathrm{~h}$ basal and IL-1 $\beta$ mediated production of PGE2, as measured by ELISA. ${ }^{* *}=p<0.01$ compared to non-stimulated control $(n=6)$. (C) Sustained knockdown of MALAT1 expression in OA osteoblasts over 21 days following repeated transfection with a MALAT1 LNA or control LNA. ${ }^{*}=p<0.05,{ }^{* * *}=p<0.001$ compared to non-stimulated control $(n=6)$. (D) Production of OPG $(\mathrm{pg} / \mathrm{mL})$ over 21 days in control LNA and MALAT1 LNA transfected OA osteoblasts $(n=6)$ as determined by ELISA. (E) Alkaline phosphatase (ALP) activity over 21 days in control LNA and MALAT1 LNA transfected OA osteoblast lysates $(n=6)$. (F) Relative amount of osteoblast mineralisation following 21 days of transfection with control (NC) or MALAT1 (M1) LNA as determined quantification of absorbance at following alizarin red staining, and representative light microscope images $(\times 40$ magnification) showing alizarin red stained mineralised bone nodules.

\section{Discussion}

This paper reports the expression and functional role of the long non-coding RNA MALAT1 in OA patient subchondral bone tissue and primary OA osteoblasts. MALAT1 was found to be highly expressed (compared to non-OA hip) in the subchondral bone tissue of both knee and hip OA patients irrespective of disease severity. On average MALAT1 expression was greater in the bone tissue from patients with greater OA severity, as measured by either KL grade or joint space narrowing. However, it should be noted that all the OA patients in this study had advanced end-stage disease, with radiographic severity determined to be either KL3 or KL4, and most patients having $<1 \mathrm{~mm}$ joint space. Therefore, we cannot comment on whether expression of MALAT1 would differ in the subchondral bone of patients with early OA.

Similar to previous findings on the role of MALAT1 in OA synovial fibroblasts [23], MALAT1 expression in OA osteoblasts was induced during the IL- $1 \beta$ inflammatory response. This suggests MALAT1 may play an important regulatory role in bone homeostasis 
under the inflammatory conditions exhibited in OA patients. Of note, although the subchondral bone expression of MALAT1 was not related to the serum concentration of IL-1 $\beta$ we did observe positive correlations between MALAT1 expression and the serum concentration of both DKK1 and galectin 1. DKK1 is an endogenous inhibitor of the Wnt/beta-catenin signalling pathway and is implicated in bone development and in the pathological remodelling of bone in both OA and osteoporosis and mediating inflammation-induced bone loss by inhibiting osteoblast differentiation [30,31]. In osteoporosis patients, serum levels of DKK1 are negatively associated with bone mineral density in the femoral head and lumbar spine [32]. In contrast, galectin-1 has been identified as secreted protein which promotes osteoblast differentiation [33], and in mediating osteoclast activity in osteolytic bone disease [34].

LNA-mediated knockdown of MALAT1 in primary human OA osteoblasts profoundly affected the transcriptomic phenotype with pathway analysis revealing significant activation of pathways that promote the production of inflammatory prostacyclins and eicosanoids. Amongst the most differentially expressed upregulated genes were TNFSF12 (>11-fold upregulated), which encodes TWEAK (TNF-related weak inducer of apoptosis). TWEAK is a known mediator of inflammatory bone remodelling [35], and targeted inhibition of the TWEAK/fibroblast growth factor inducible 14 (FnF14) signalling pathway has been proposed as a therapeutic strategy to reduce bone resorption in rheumatoid arthritis patients [36]. In addition, osteoblasts depleted of MALAT1 exhibited a significant $>10$-fold upregulation in the expression of PTGS2, the gene which encodes for the enzyme COX2 that mediates the production of inflammatory prostaglandins including the putative OA pain mediator PGE2 $[37,38]$. Indeed, we found that both basal and IL-1 $\beta$ induced PGE2 secretion was significantly greater in osteoblasts depleted of MALAT1 compared to control cells. PGE2 acting through the E prostanoid receptors EP2 and EP4 sensitizes nociceptors, possibly by acting synergistically with IL1 $\beta$ to induce IL-6 and $i N O S$ expression [39] and selective COX2 inhibitors such as celecoxib are known to reduce pain and inflammation in OA patients [40]. The subchondral bone is considered to be an important site of OA pain, being innervated with sensory neurones [41] and bone marrow lesions (identified as hyper-intense regions on T1-weighted MRI scans) and bone shape in OA patients being associated with bone pain [42]. Therefore, our finding here that MALAT1 expression in OA osteoblasts regulates PTGS2 expression as well as both basal and IL-1 $\beta$ induced PGE2 production suggests that MALAT1 may play an important role in regulating inflammatory pain in the bone.

Despite the effect of MALAT1 knockdown on the osteoblast transcriptome and the acute effect on PGE2 production we did not observe any chronic effect of MALAT1 knockdown on several key osteoblast functions. During a time-period of 3 weeks, we induced sustained MALAT1 knockdown but did not observe any significant difference in the secretion of OPG or in the activity of ALP and ability of the osteoblast to form mineralised bone nodules. This contrasts with previous publications which have implicated MALAT1 in mediating both OPG production in osteoblasts [43] as well as ALP activity and mineralisation during osteoblast differentiation [44].

The underlining molecular mechanisms by which lncRNAs mediate their function is complex and for the majority of lncRNAs remains to be determined. Several lncRNAs, including MALAT1 have been reported to exert a pro-osteogenic function by acting as miRNA sponges [45]. Indeed, the reported role for MALAT1 in mediating osteoblast differentiation has been linked to the sponging of several miRNAs including miR-204 [45], $\mathrm{miR}-30$ [26] and miR-43 [46]. In our analysis of the OA osteoblast transcriptome analysis following MALAT1 depletion, we did not identify any miRNAs which were altered upon MALAT1 KD. However, the isolation of total RNA using columns would likely have excluded many miRNAs from our sequencing analysis. 


\section{Conclusions}

In conclusion, the expression of MALTA1 in OA subchondral bone, its induction in osteoblasts upon inflammatory challenge and its functional role in modulating the production of the prostaglandin PGE2 suggest that MALAT1 may play an important role in the development of $\mathrm{OA}$ bone pain and inflammation.

Supplementary Materials: The following are available online at https:/ / www.mdpi.com/article/10 .3390/cells10040786/s1, Table S1: MALAT1 and joint severity, Table S2: RNAseq All DEGs, Table S3: RNAseq Common DEGs.

Author Contributions: Conceptualization S.W.J. and F.A.A. Methodology and analysis F.A.A., A.S.-O., S.N.W., D.E.N., H.F., T.N., E.T.D. and S.W.J. Writing of original draft, review and editing S.W.J. and F.A.A. All authors have read and agreed to the published version of the manuscript.

Funding: This study was partially funded by the Kuwait Foundation for the Advancement of Sciences (KFAS; CB18-63MB-0).

Institutional Review Board Statement: The study was conducted according to the guidelines of the Declaration of Helsinki and approved by the UK National Research Ethics Committee (South East Scotland Research Ethics Committee, UK; reference NRES 16/SS/0172 and NRES 14/ES/1044; date of approval 31/10/2016 and 12/08/2014).

Informed Consent Statement: Informed consent was obtained from all subjects involved in the study.

Data Availability Statement: The data discussed in this publication have been deposited in NCBI's Gene Expression Omnibus and are accessible through GEO Series accession number GSE167918. (https: / / www.ncbi.nlm.nih.gov / geo / query / acc.cgi?acc=GSE167918; deposited on 28 February 2021).

Conflicts of Interest: The authors declare no conflict of interest. The funders had no role in the design, execution, interpretation, or writing of the study.

\section{References}

1. Philp, A.M.; Davis, E.T.; Jones, S.W. Developing anti-inflammatory therapeutics for patients with osteoarthritis. Rheumatology 2017, 56, 869-881. [CrossRef] [PubMed]

2. Tonge, D.P.; Pearson, M.J.; Jones, S.W. The hallmarks of osteoarthritis and the potential to develop personalised disease-modifying pharmacological therapeutics. Osteoarthr. Cartil. 2014, 22, 609-621. [CrossRef] [PubMed]

3. Hawker, G.A. Osteoarthritis is a serious disease. Clin. Exp. Rheumatol. 2019, 37, 3-6. [PubMed]

4. Primorac, D.; Molnar, V.; Rod, E.; Jelec, Z.; Cukelj, F.; Matisic, V.; Vrdoljak, T.; Hudetz, D.; Hajsok, H.; Boric, I. Knee Osteoarthritis: A Review of Pathogenesis and State-Of-The-Art Non-Operative Therapeutic Considerations. Genes 2020, 11. [CrossRef]

5. Dibonaventura, M.; Gupta, S.; McDonald, M.; Sadosky, A. Evaluating the health and economic impact of osteoarthritis pain in the workforce: Results from the National Health and Wellness Survey. BMC Musculoskelet. Disord. 2011, 12, 83. [CrossRef]

6. Hunter, D.J.; Gerstenfeld, L.; Bishop, G.; Davis, A.D.; Mason, Z.D.; Einhorn, T.A.; Maciewicz, R.A.; Newham, P.; Foster, M.; Jackson, S.; et al. Bone marrow lesions from osteoarthritis knees are characterized by sclerotic bone that is less well mineralized. Arthritis Res. Ther. 2009, 11, R11. [CrossRef]

7. Philp, A.M.; Collier, R.L.; Grover, L.M.; Davis, E.T.; Jones, S.W. Resistin promotes the abnormal Type I collagen phenotype of subchondral bone in obese patients with end stage hip osteoarthritis. Sci. Rep. 2017, 7, 4042. [CrossRef]

8. Bailey, A.J.; Sims, T.J.; Knott, L. Phenotypic expression of osteoblast collagen in osteoarthritic bone: Production of type I homotrimer. Int. J. Biochem. Cell Biol. 2002, 34, 176-182. [CrossRef]

9. Burr, D.B.; Gallant, M.A. Bone remodelling in osteoarthritis. Nat. Rev. Rheumatol. 2012, 8, 665-673. [CrossRef]

10. Chang, J.; Jackson, S.G.; Wardale, J.; Jones, S.W. Hypoxia modulates the phenotype of osteoblasts isolated from knee osteoarthritis patients, leading to undermineralized bone nodule formation. Arthritis Rheumatol. 2014, 66, 1789-1799. [CrossRef]

11. Quasnichka, H.L.; Anderson-MacKenzie, J.M.; Bailey, A.J. Subchondral bone and ligament changes precede cartilage degradation in guinea pig osteoarthritis. Biorheology 2006, 43, 389-397.

12. Radin, E.L.; Rose, R.M. Role of subchondral bone in the initiation and progression of cartilage damage. Clin. Orthop. Relat. Res. 1986, 213, 34-40. [CrossRef]

13. Khalil, A.M.; Guttman, M.; Huarte, M.; Garber, M.; Raj, A.; Rivea Morales, D.; Thomas, K.; Presser, A.; Bernstein, B.E.; van Oudenaarden, A.; et al. Many human large intergenic noncoding RNAs associate with chromatin-modifying complexes and affect gene expression. Proc. Natl. Acad. Sci. USA 2009, 106, 11667-11672. [CrossRef]

14. Wang, K.C.; Chang, H.Y. Molecular mechanisms of long noncoding RNAs. Mol. Cell 2011, 43, 904-914. [CrossRef] 
15. Ilott, N.E.; Heward, J.A.; Roux, B.; Tsitsiou, E.; Fenwick, P.S.; Lenzi, L.; Goodhead, I.; Hertz-Fowler, C.; Heger, A.; Hall, N.; et al. Corrigendum: Long non-coding RNAs and enhancer RNAs regulate the lipopolysaccharide-induced inflammatory response in human monocytes. Nat. Commun. 2015, 6, 6814. [CrossRef]

16. Roux, B.T.; Heward, J.A.; Donnelly, L.E.; Jones, S.W.; Lindsay, M.A. Catalog of Differentially Expressed Long Non-Coding RNA following Activation of Human and Mouse Innate Immune Response. Front. Immunol. 2017, 8, 1038. [CrossRef]

17. Wijesinghe, S.N.; Nicholson, T.; Tsintzas, K.; Jones, S.W. Involvements of long noncoding RNAs in obesity-associated inflammatory diseases. Obes. Rev. 2020. [CrossRef]

18. Hadjicharalambous, M.R.; Roux, B.T.; Feghali-Bostwick, C.A.; Murray, L.A.; Clarke, D.L.; Lindsay, M.A. Long Non-coding RNAs Are Central Regulators of the IL-1beta-Induced Inflammatory Response in Normal and Idiopathic Pulmonary Lung Fibroblasts. Front. Immunol. 2018, 9, 2906. [CrossRef]

19. Hadjicharalambous, M.R.; Lindsay, M.A. Idiopathic Pulmonary Fibrosis: Pathogenesis and the Emerging Role of Long NonCoding RNAs. Int. J. Mol. Sci. 2020, 21, 524. [CrossRef]

20. Hamann, P.D.; Roux, B.T.; Heward, J.A.; Love, S.; McHugh, N.J.; Jones, S.W.; Lindsay, M.A. Transcriptional profiling identifies differential expression of long non-coding RNAs in Jo-1 associated and inclusion body myositis. Sci. Rep. 2017, 7, 8024. [CrossRef]

21. Pearson, M.J.; Jones, S.W. Review: Long Noncoding RNAs in the Regulation of Inflammatory Pathways in Rheumatoid Arthritis and Osteoarthritis. Arthritis Rheumatol. 2016, 68, 2575-2583. [CrossRef]

22. Pearson, M.J.; Philp, A.M.; Heward, J.A.; Roux, B.T.; Walsh, D.A.; Davis, E.T.; Lindsay, M.A.; Jones, S.W. Long Intergenic Noncoding RNAs Mediate the Human Chondrocyte Inflammatory Response and Are Differentially Expressed in Osteoarthritis Cartilage. Arthritis Rheumatol. 2016, 68, 845-856. [CrossRef]

23. Nanus, D.E.; Wijesinghe, S.N.; Pearson, M.J.; Hadjicharalambous, M.R.; Rosser, A.; Davis, E.T.; Lindsay, M.A.; Jones, S.W. Regulation of the Inflammatory Synovial Fibroblast Phenotype by Metastasis-Associated Lung Adenocarcinoma Transcript 1 Long Noncoding RNA in Obese Patients With Osteoarthritis. Arthritis Rheumatol. 2020, 72, 609-619. [CrossRef]

24. Yang, X.; Zhang, Y.; Li, Y.; Wen, T. MALAT1 enhanced the proliferation of human osteoblasts treated with ultrahigh molecular weight polyethylene by targeting VEGF via miR225p. Int. J. Mol. Med. 2018, 41, 1536-1546. [CrossRef]

25. Zhu, J.; Zhang, X.; Gao, W.; Hu, H.; Wang, X.; Hao, D. lncRNA/circRNAmiRNAmRNA ceRNA network in lumbar intervertebral disc degeneration. Mol. Med. Rep. 2019, 20,3160-3174. [CrossRef]

26. Yi, J.; Liu, D.; Xiao, J. LncRNA MALAT1 sponges miR-30 to promote osteoblast differentiation of adipose-derived mesenchymal stem cells by promotion of Runx2 expression. Cell Tissue Res. 2019, 376, 113-121. [CrossRef]

27. Wang, Q.; Yang, Q.; Chen, G.; Du, Z.; Ren, M.; Wang, A.; Zhao, H.; Li, Z.; Zhang, G.; Song, Y. LncRNA expression profiling of BMSCs in osteonecrosis of the femoral head associated with increased adipogenic and decreased osteogenic differentiation. Sci. Rep. 2018, 8, 9127. [CrossRef] [PubMed]

28. Dobin, A.; Davis, C.A.; Schlesinger, F.; Drenkow, J.; Zaleski, C.; Jha, S.; Batut, P.; Chaisson, M.; Gingeras, T.R. STAR: Ultrafast universal RNA-seq aligner. Bioinformatics 2013, 29, 15-21. [CrossRef]

29. Love, M.I.; Huber, W.; Anders, S. Moderated estimation of fold change and dispersion for RNA-seq data with DESeq2. Genome Biol. 2014, 15, 550. [CrossRef]

30. Qiang, Y.W.; Barlogie, B.; Rudikoff, S.; Shaughnessy, J.D., Jr. Dkk1-induced inhibition of Wnt signaling in osteoblast differentiation is an underlying mechanism of bone loss in multiple myeloma. Bone 2008, 42, 669-680. [CrossRef] [PubMed]

31. Hurson, C.J.; Butler, J.S.; Keating, D.T.; Murray, D.W.; Sadlier, D.M.; O’Byrne, J.M.; Doran, P.P. Gene expression analysis in human osteoblasts exposed to dexamethasone identifies altered developmental pathways as putative drivers of osteoporosis. BMC Musculoskelet. Disord. 2007, 8, 12. [CrossRef] [PubMed]

32. Butler, J.S.; Murray, D.W.; Hurson, C.J.; O’Brien, J.; Doran, P.P.; O’Byrne, J.M. The role of Dkk1 in bone mass regulation: Correlating serum Dkk1 expression with bone mineral density. J. Orthop. Res. 2011, 29, 414-418. [CrossRef] [PubMed]

33. Andersen, H.; Jensen, O.N.; Moiseeva, E.P.; Eriksen, E.F. A proteome study of secreted prostatic factors affecting osteoblastic activity: Galectin-1 is involved in differentiation of human bone marrow stromal cells. J. Bone Miner. Res. 2003, 18, 195-203. [CrossRef] [PubMed]

34. Muller, J.; Duray, E.; Lejeune, M.; Dubois, S.; Plougonven, E.; Leonard, A.; Storti, P.; Giuliani, N.; Cohen-Solal, M.; Hempel, U.; et al Loss of Stromal Galectin-1 Enhances Multiple Myeloma Development: Emphasis on a Role in Osteoclasts. Cancers 2019, 11, 261. [CrossRef]

35. Vincent, C.; Findlay, D.M.; Welldon, K.J.; Wijenayaka, A.R.; Zheng, T.S.; Haynes, D.R.; Fazzalari, N.L.; Evdokiou, A.; Atkins, G.J. Pro-inflammatory cytokines TNF-related weak inducer of apoptosis (TWEAK) and TNFalpha induce the mitogen-activated protein kinase (MAPK)-dependent expression of sclerostin in human osteoblasts. J. Bone Miner. Res. 2009, 24, 1434-1449. [CrossRef]

36. Du, Y.Y.; Zhao, Y.X.; Liu, Y.P.; Liu, W.; Wang, M.M.; Yuan, C.M. Regulatory Tweak/Fn14 signaling pathway as a potent target for controlling bone loss. Biomed. Pharmacother. 2015, 70, 170-173. [CrossRef]

37. Yao, C.; Narumiya, S. Prostaglandin-cytokine crosstalk in chronic inflammation. Br. J. Pharmacol. 2019, 176, 337-354. [CrossRef]

38. Lee, A.S.; Ellman, M.B.; Yan, D.; Kroin, J.S.; Cole, B.J.; van Wijnen, A.J.; Im, H.J. A current review of molecular mechanisms regarding osteoarthritis and pain. Gene 2013, 527, 440-447. [CrossRef]

39. Li, X.; Ellman, M.; Muddasani, P.; Wang, J.H.; Cs-Szabo, G.; van Wijnen, A.J.; Im, H.J. Prostaglandin E2 and its cognate EP receptors control human adult articular cartilage homeostasis and are linked to the pathophysiology of osteoarthritis. Arthritis Rheum. 2009, 60, 513-523. [CrossRef] 
40. Puljak, L.; Marin, A.; Vrdoljak, D.; Markotic, F.; Utrobicic, A.; Tugwell, P. Celecoxib for osteoarthritis. Cochrane Database Syst. Rev. 2017, 5, CD009865. [CrossRef]

41. Suri, S.; Gill, S.E.; Massena de Camin, S.; Wilson, D.; McWilliams, D.F.; Walsh, D.A. Neurovascular invasion at the osteochondral junction and in osteophytes in osteoarthritis. Ann. Rheum. Dis. 2007, 66, 1423-1428. [CrossRef]

42. Barr, A.J.; Campbell, T.M.; Hopkinson, D.; Kingsbury, S.R.; Bowes, M.A.; Conaghan, P.G. A systematic review of the relationship between subchondral bone features, pain and structural pathology in peripheral joint osteoarthritis. Arthritis Res. Ther. 2015, 17, 228. [CrossRef]

43. Che, W.; Dong, Y.; Quan, H.B. RANKL inhibits cell proliferation by regulating MALAT1 expression in a human osteoblastic cell line hFOB 1.19. Cell Mol. Biol. 2015, 61, 7-14.

44. Xiao, X.; Zhou, T.; Guo, S.; Guo, C.; Zhang, Q.; Dong, N.; Wang, Y. LncRNA MALAT1 sponges miR-204 to promote osteoblast differentiation of human aortic valve interstitial cells through up-regulating Smad4. Int. J. Cardiol. 2017, 243, 404-412. [CrossRef]

45. Silva, A.M.; Moura, S.R.; Teixeira, J.H.; Barbosa, M.A.; Santos, S.G.; Almeida, M.I. Long noncoding RNAs: A missing link in osteoporosis. Bone Res. 2019, 7, 10. [CrossRef]

46. Gao, Y.; Xiao, F.; Wang, C.; Wang, C.; Cui, P.; Zhang, X.; Chen, X. Long noncoding RNA MALAT1 promotes osterix expression to regulate osteogenic differentiation by targeting miRNA-143 in human bone marrow-derived mesenchymal stem cells. J. Cell Biochem. 2018, 119, 6986-6996. [CrossRef] 